\title{
Kentron
}

REVUE PLURIDISCIPL

Revue pluridisciplinaire du monde antique

19 | 2003

Le statut et l'image du corps dans la mythologie et la littérature grecques (suite et fin)

\section{Créatures composites en Mésopotamie}

\section{Christine Dumas-Reungoat}

\section{(2) OpenEdition \\ 1 Journals}

\section{Édition électronique}

URL : https://journals.openedition.org/kentron/1852

DOI : $10.4000 /$ kentron. 1852

ISSN : 2264-1459

\section{Éditeur}

Presses universitaires de Caen

\section{Édition imprimée}

Date de publication : 31 décembre 2003

Pagination : 91-113

ISBN : 2-84133-222-5

ISSN : 0765-0590

\section{Référence électronique}

Christine Dumas-Reungoat, "Créatures composites en Mésopotamie », Kentron [En ligne], 19| 2003,

mis en ligne le 12 avril 2018, consulté le 13 octobre 2022. URL : http://journals.openedition.org/

kentron/1852 ; DOI : https://doi.org/10.4000/kentron.1852

\section{(c)}

Creative Commons - Attribution - Pas d'Utilisation Commerciale - Pas de Modification 4.0 International - CC BY-NC-ND 4.0

https://creativecommons.org/licenses/by-nc-nd/4.0/ 


\section{CRÉATURES COMPOSITES EN MÉSOPOTAMIE}

Nous connaissons tous les créatures étranges qui peuplent les récits de la mythologie grecque et qui sont l'objet de nombreuses représentations iconographiques, ces êtres hybrides, mi-hommes, mi-animaux. Citons comme exemples la Chimère, le Griffon, la Gorgone, la Sirène ou la Sphinge ${ }^{1}$. Or, comme dans beaucoup de domaines, la Grèce a adapté des données qui lui étaient étrangères et se les est appropriées pour créer des synthèses originales - n'oublions jamais que la Grèce est pour partie sise en Orient et qu'elle fait partie de l'Asie, pour reprendre l'expression de Martin L. West ${ }^{2}$, et que les habitants de l'ancienne Mésopotamie ne sont pas de très lointains voisins pour elle, dans la mesure où des peuples comme les Hittites et les Hourrites ont servi de relais entre ces peuples. Il serait intéressant de voir quelles créatures composites imaginées par les anciens Sumériens et Akkadiens ont pu nourrir l'imaginaire des Grecs. Car, en matière de monstres, la Mésopotamie n'a rien à envier à la Grèce : combien d'êtres hybrides les anciens habitants de la Mésopotamie n’ont-ils pas inventés! Combien n'en ont-ils pas représentés sur des sceaux-cylindres, décrits dans des épopées ou visions de rêve ou de cauchemar, postés aux portes de leurs palais? En faire l'inventaire nécessiterait de très longs développements, il n'empêche que les regarder chacun tour à tour en détail ne manquerait pas d'intérêt tant les variantes d'un être composite à l'autre sont nombreuses et étonnantes. Tentons donc d'en établir une classification pour voir un peu plus clair dans cette forêt de monstres et mettre en évidence quels points communs ces créatures composites peuvent avoir avec celles de la Grèce ancienne, en faisant un détour par le monde hittite qui offre également des hybrides très surprenants.

\section{Classification des créatures composites en Mésopotamie}

Un premier contact avec les monstres de la Mésopotamie donne l'impression d'un monde étrange et effrayant, ensemble confus de créatures fantastiques ${ }^{3}$. Toutefois,

1. Pour une liste des hybrides grecs, voir en annexe I de l'article le tableau où nous avons rassemblé les créatures composites grecques, sans avoir la prétention d'être exhaustive; pour les sources littéraires de ces figures, se reporter aux références aux auteurs anciens données dans les notes aux différentes entrées du dictionnaire de P. Grimal (Grimal 1951).

2. West 1960, 31.

3. Pour un aperçu des principales figures, cf. annexe II de ce travail. 
en regardant de plus près les créatures hybrides décrites dans les récits mythologiques et celles qui figurent dans la glyptique et les décors des temples et palais, l'on parvient à distinguer un certain ordre dans ce monde foisonnant. Voici trois pistes de classement: selon leurs particularités morphologiques, leur nature ou leur(s) fonction(s).

\section{Classification des créatures composites selon des critères morphologiques}

D'un point de vue morphologique, on peut remarquer tout d'abord que les êtres hybrides sont tous composés d'éléments humains et animaux, à une exception près qui fait intervenir un élément inanimé : le dieu-bateau, représenté sur un cylindre de l'époque présargonique (2800-2470 av. J.-C. $\left.{ }^{4}\right)$ comme un homme à tête barbue, muni de bras, et dont le buste se prolonge en forme de bateau ${ }^{5}$. On rencontre ce motif dans le cadre de poèmes célébrant des voyages et processions de dieux. Le dieu du Soleil, Shamash, par exemple, est représenté dans sa barge anthropomorphisée, transportant ses objets rituels et symboles ${ }^{6}$. L'homme-bateau est le rameur qui fait avancer l'embarcation tandis que le dieu tient également une rame qui apparemment sert de gouvernail.

Pour le choix des animaux, il n'est en soi pas très original, ce sont les combinaisons qui le sont. On trouve une quinzaine de représentants de la faune sauvage et domestique de la Mésopotamie que nous pouvons énumérer : lion, scorpion, aigle, mouflon, bouquetin, serpent (/ lézard), taureau (/ bison), chèvre, poisson, oiseau, bœuf, âne et cheval. Les combinaisons peuvent rassembler des éléments anatomiques humains et animaux ou bien seulement des éléments propres à différents animaux. Ainsi deux groupes se dessinent : l'un à dominante humaine dont les figures sont en station verticale, l'autre à dominante animale dont les représentations sont en station horizontale.

Dans la composition des hybrides, trois procédés apparaissent :

- le premier, l'adjonction d'éléments appartenant à une autre espèce: cornes, oreilles, queue, ailes : pour illustrer ce procédé, citons les taureaux et lions androcéphales ailés, le griffon, le dragon-serpent, le mouflon ailé, l'aigle léontocéphale (Imdugud), le sphinx ailé à tête de bélier...;

- le deuxième, la juxtaposition d'éléments:

- homme / animal: et ce sont les sirènes, tritons, centaure, lion-centaure, hommescorpion;

4. Toutes les dates et mentions de siècles dans ce travail se réfèrent à l'époque antérieure à notre ère, nous ne le précisons pas systématiquement, mais il faut les comprendre avec le signe "moins".

5. Parrot 1960, 193 et 165, fig. 238 et fig. 198.

6. Black \& Green 1992, 45. 
- animal / animal : le poisson-lion et le poisson-chèvre fournissent deux exemples fort originaux;

- homme / inanimé : c'est le cas du bateau anthropomorphisé;

- le troisième consiste en la déformation morphologique faite dans un but décoratif ou disons qu'il s'agit des anomalies dues à une convention stylistique. Le meilleur exemple est le taureau ailé à tête humaine et barbue de plus de quatre mètres de hauteur, qui est posté comme gardien de la porte monumentale à l'entrée du palais royal du roi assyrien Sargon II (dont le règne a duré de 721 à 705 avant notre ère) dans l'actuelle Khorsabad. Cette créature hybride était destinée à être vue aussi bien de face que de côté, aussi est-elle dotée de cinq pattes (peut-être l'un des plus vieux trompe-l'œil de l'histoire! $)^{7}$.

\section{Classification des créatures composites en fonction de leur nature: être supranaturel, démon ou monstre}

La nature des créatures composites en Mésopotamie est la plupart du temps d'ordre surnaturel. On trouve ainsi des génies, divinités mineures, appartenant au personnel des cours célestes, puisqu'à l'image des rois, les dieux avaient aussi leur cour. Ce sont des êtres hybrides qui peuvent se présenter sous la forme d'êtres humains ailés, coiffés ou non d'un bonnet à cornes, signe de leur caractère divin, et selon les cas pourvus d'une tête animale, comme dans le cas du génie-griffon ${ }^{8}$ dont la tête est celle d'un oiseau. Certains êtres hybrides sont les symboles des dieux. Ainsi, Marduk, durant la période paléo-babylonienne, s'attribue le dragon-serpent (mushhushshu), animal emblématique de Tishpak, le dieu suprême d'Eshnunna, capitale du pays de Wârum à l'époque paléo-babylonienne. Cet animal étrange est une sorte de dragon cornu, ayant le corps et la tête d'un serpent, les pattes antérieures d'un lion, les pattes postérieures d'un oiseau. Cet hybride est parfois simplement un être magiquement protecteur, sans être associé de façon spécifique à une quelconque divinité. C'est le cas également du poisson-chèvre qui peut être le symbole d'Éa (Enki, en sumérien), dieu des eaux.

Dans leur dictionnaire sur les dieux, démons et symboles de Mésopotamie, J. Black et A. Green, à l'article «Demons and Monsters », rappellent au sujet des créatures composites les dénominations qu'on leur applique. D’abord, précisons que certains personnages sont parfois présentés comme des dieux et à d'autres moments comme des génies: une certaine confusion règne entre les deux niveaux. De façon générale, les créatures composites sont plus rarement de nature divine: génies et monstres l'emportent dans ce domaine. Dans les études modernes de l'art et de

7. Ibid., 50, pour une représentation de cet hybride.

8. Pour deux représentations de cet hybride, cf. ibid., 99 et 100. 
l'iconographie de l'ancienne Mésopotamie, le terme demon a généralement été appliqué à n'importe quelle créature hybride debout, pourvue d'un corps humain, tandis que monster est appliqué à un être résultant d'une combinaison d'éléments animaux et reposant sur quatre pattes. Toutefois, précisent les auteurs de l'article, dans les textes anciens, ce sont les termes râbisu en akkadien et mashkim en sumérien, qui désignent des êtres supranaturels se situant entre le niveau des dieux et celui des hommes, que l'on peut traduire en grec approximativement par $\delta \alpha i \mu \omega v$, mot qui dans son sens originel désigne justement un être supranaturel, un esprit. Et J. Black

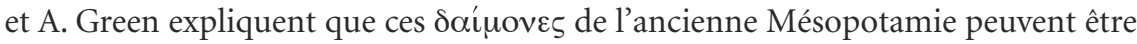
bons ou mauvais, selon la qualification qui leur est attribuée. Ils figurent plus rarement dans les récits mythologiques que dans les incantations magiques. Généralement ceux qui sont mauvais sont les simples exécuteurs des volontés divines, c'est leur rôle essentiel : ils appliquent le châtiment ordonné par les dieux en expiation d'une faute. Et de tels mauvais génies étaient souvent imaginés comme des « esprits météorologiques ", du vent ou des tempêtes, principalement. Leur méthode habituelle pour s'attaquer au genre humain consistait à leur infliger des maladies, même si on ne considérait pas qu'ils étaient à l'origine de toutes les maladies. De façon générale, on représentait assez rarement mauvais dieux et mauvais génies: peut-être redoutait-on alors quelque malheur à la simple vue de leur image... Pourtant, autour du premier millénaire avant notre ère Lamashtu était représentée de façon courante dans le cadre d'incantations à son encontre. Ses principales victimes étaient les bébés à naître et les nouveau-nés. Cet être supranaturel, mauvais, était particulièrement effrayant : tête de lion, dents et parfois oreilles d'âne, poitrine dénudée, corps velu, mains souillées (de sang?), doigts et ongles longs, et ses pieds semblables à ceux d'Anzû (Imdugud en sumérien), c'est-à-dire à des serres d'oiseau. Enfin elle tient des serpents dans ses mains. Un autre génie (ou dieu) souterrain, bienveillant celuici - il protège les hommes des vents d'Ouest pestilentiels et les femmes enceintes quand elles portent autour du cou des amulettes le représentant -, Pazuzu (qui a une tête plutôt semblable à celle d'un chien, des yeux anormalement protubérants, un corps couvert d'écailles, un sexe à tête de serpent, des serres d'oiseau et des ailes), est montré forçant Lamashtu à descendre dans le monde souterrain : c'est qu'à partir du premier millénaire, on imaginait le monde souterrain peuplé de ces mauvais génies.

Cette dernière remarque nous invite à rappeler une chronologie très simplifiée, mais plausible, que proposent J. Black et A. Green ${ }^{9}$, pour rendre compte du développement des génies et des monstres dans l'art de l'ancienne Mésopotamie. Cette chronologie comporte cinq phases:

9. Black \& Green 1992, 63. Nous traduisons librement cette partie de l'article concernant la chronologie du développement des monstres et êtres supranaturels composites. 
1- Une phase de formation dans les périodes tardives d'Obeid (6500-3700 / 4000) et d'Uruk (3700-3400), quand les traits caractéristiques de différents animaux furent pour les premières fois combinés, donnant le jour à des êtres hybrides jamais vus dans la nature.

2- Une phase "optimiste», au cours de la période d'Akkad (2350-2000), lorsque les scènes de glyptique montrent la capture et le châtiment des génies infâmes.

3- Une phase "équilibrée ", au cours de la période paléo-babylonienne (20001600 ) où les motifs sur les sceaux-cylindres présentent souvent des images (dieux, symboles et autres motifs s'y côtoient) mêlant bons et mauvais éléments concernant les hommes.

4- Une phase de "transformation», avec l'art mitannien, cassite et médio-assyrien du XIv ${ }^{e}$ au XI ${ }^{e}$ siècle avant notre ère, quand l'imagerie de la période précédente, centrée davantage sur l'homme, laisse place aux hybrides à tête animale, qui deviennent prépondérants.

5- Une phase qualifiée de «demonic» par les auteurs de cette étude, représentée par l'art néo-assyrien et néo-babylonien (900-550), quand les mauvais génies, particularisés, sont dépeints dans leur horreur complète. Cette dernière phase s'accorde bien avec la nouvelle théologie du $\mathrm{I}^{\mathrm{er}}$ millénaire d'un monde souterrain habité par eux. Ce changement apparaît, qui plus est, au moment même où dans les palais et temples sont érigées des statues monumentales d'êtres - magiquement - protecteurs et où l'on pratique l'ensevelissement, dans les fondations de ces édifices, de petites représentations en argile de ces êtres protecteurs. Et dans les récits mythologiques, les génies et monstres finissent par être considérés comme un groupe à cette époque.

Or précisément, parmi les tablettes qui nous sont parvenues d'Assur, l'une, vraisemblablement écrite au cours du viI ${ }^{\mathrm{e}}$ siècle avant notre ère,

renferme une des plus intéressantes et des plus pittoresques évocations que nous ayons du monde infernal, tel qu'à cette époque on se l'imaginait sans doute en Assyrie,

explique René Labat, dans la présentation de la traduction qu'il en propose ${ }^{10}$. Peutêtre poussé par le désespoir devant le malheur que connaît son règne, le roi Kummâ (inconnu par ailleurs) paraît avoir souhaité « descendre en Enfer» (1. 28), expression dont les traducteurs ne sont pas sûrs qu'il faille l'entendre à la lettre plutôt que comme une façon de marquer le désir de la mort ${ }^{11}$. Pour obtenir cette grâce, il fait sacrifices et prières, invoquant notamment Allatu, la «Dame de l'Enfer» (1. 29), autre nom de la Reine infernale Ereshkigal. Et vient le jour où son vœu imprudent se réalise, mais en songe; et il est censé raconter lui-même cette vision nocturne (1. 41), d'où

10. Labat 1970, 94 .

11. Cf. Bottéro 1972-1973, 94. 
le récit à la première personne du singulier, pour décrire d'abord la cour des seize hauts personnages divins et démoniaques qui entourent et secondent le roi et la reine d'Enfer. Citons-en quelques-uns : Namtar : «Destin », «Lieutenant de l'Enfer, l'Exécuteur des commandements»; Namtartu: «Destinée», son épouse; Mûtu: «Trépas»; Shêdu-Lemnu : «Méchant Diable »; Alluhappu : « Filet-de-chasse », dans lequel les hommes sont empêtrés et pris par la Mort; Humut-tabal: «Emporte-Vite», le Nocher de l'Enfer, «Et bien d'autres que le narrateur avoue ne pas toujours reconnaître, comme pour souligner que l'Enfer est plus riche que nous n'en pouvons savoir ${ }^{12}$. Tous ces êtres surnaturels sont décrits par celui qui est censé les avoir contemplés: ce sont des créatures composites, dix d'entre elles au moins ont des mains ou des pieds humains, un a une tête d'homme, c'est Shêdu; les animaux entrant dans ces compositions sont le lion, l'oiseau, le dragon, le bœuf, la chèvre et l'oiseau Anzû : il s'agit d'un aigle colossal à tête de lion, dont le battement des ailes provoquait la tempête. Les détails anatomiques concernent la tête (coiffée d'une tiare ou non, le nombre de têtes), les mains et les pieds ou pattes (dont il donne le nombre) ainsi que les ailes. Le plus effrayant de tous est peut-être celui dont nous n'avons que la dernière syllabe du nom, « $[\ldots] \mathrm{ra} »:$ « [...] ra avait trois pattes antérieures d'oiseau, une postérieure de bœuf; il avait une terrifiante aura lumineuse ${ }^{13}$. Jean Bottéro précise au sujet de cette vision infernale particulièrement effrayante, composée dans un style «assez artificiel et ampoulé », que:

Nous connaissons ce genre de tableaux par quelques documents qui décrivent, en fait des statues, figurines ou reliefs ${ }^{14}$, mais, dans le présent contexte, il est vraisemblable que l'imagination des auteurs s'est déchaînée pour donner de la cour infernale un tableau propre à frapper d'effroi ${ }^{15}$.

Et pour souligner nettement quelle forte angoisse a saisi Kummâ à son réveil, les auteurs multiplient les comparaisons pleines d'emphase, conformément au goût du temps. Il s'agit sûrement d'un moyen stylistique pour traduire la valeur accordée aux rêves par les anciens Mésopotamiens: pour eux, l'expérience onirique n'était pas illusoire comme pour nous, mais tout aussi réelle, à sa façon, que la vie de chaque jour.

Ainsi, la classification selon la nature de l'être hybride n'est pas toujours satisfaisante, puisque souvent, d'un texte à l'autre ou bien d'une époque à l'autre, le personnage peut changer de catégorie (dieu / génie, génie / symbole).

12. Nous reprenons ici les traductions proposées par J. Bottéro pour les noms figurant aux lignes 42 à 48 du texte de cette vision infernale (et sa conclusion sur cette énumération) : ibid., 95-96.

13. Labat 1970,95 .

14. Bottéro 1972-1973, 96, où est cité notamment Köcher, Fr. (1953), «Der Babylonische Göttertypentext », Mitteilungen des Instituts für Orientforschung, I, 57 sq.

15. Ibid., 96. 


\section{Classification des créatures composites selon leur fonction}

Nous pouvons également tenter d'esquisser une classification des créatures composites en tenant compte de certaines fonctions qu'elles jouent dans les récits mythiques comme dans l'art.

Avec la vision du monde infernal de Kummâ, nous venons de voir qu'une des fonctions des êtres hybrides consiste à effrayer le lecteur ou à créer une forte impression sur le spectateur, comme c'est le cas dans le cadre des palais et des temples aux portes et escaliers monumentaux. Là, les représentations d'êtres hybrides ne font que contribuer au caractère imposant des constructions, inspirant ainsi aux usagers de ces lieux le respect pour les divinités qui y reçoivent un culte ou pour la personne du roi. C'est ainsi que des lions-centaures sont placés à l'entrée des palais assyriens (à Kalakh - actuelle Nimrud - par exemple) et des taureaux androcéphales ailés aux portes de ceux de Kalakh, Ninive ou Dûr-Sharrukin - actuelle Khorsabad - où ils jouent le rôle de gardien par leur stature colossale et étrange : ce sont les êtres magiquement protecteurs des lieux. André Parrot ${ }^{16}$ expose le cas de la ville de Guzana, qui renferme beaucoup de ces monstres:

Kapara, fils de Hadianu, a décidé, en tant que monarque plein d'audace, de conférer [à Guzana] le lustre d'une capitale par des constructions imposantes ornementées de centaines de reliefs. Parmi temples et palais, se dressait une construction [...] qui abritait la plus étrange collection de sculptures qu'on puisse concevoir. Tout semblait y avoir été réuni pour plonger le visiteur dans un monde mystérieux et hallucinant. Pour arriver à la terrasse sur laquelle on avait construit l'édifice il fallait d'abord franchir un passage gardé par deux monstres ailés, androcéphales, mi-oiseaux, mi-scorpions. Puis s'offrait un spectacle peu banal quand on arrivait à la terrasse : un grand portail encadré de deux sphinx (voilés) et découpé en quatre baies par trois grandes statues caryatides (deux hommes et une femme) debout sur des animaux d'aspect peu engageant : un lion, un taureau et une lionne. Avec leurs yeux de pierre blanche incrustés d'une pastille de schiste noir, enchâssés dans la masse sombre du basalte, tous ces monstres devaient provoquer une crainte salutaire ou une épouvantable frayeur que n'apaisaient certainement pas les deux griffons ailés postés de part et d'autre de la porte donnant accès à la chambre intérieure. Celle-ci était nue, sans installation aucune qui pût assurer une identification et une attribution à un panthéon ou à un prince.

Si des êtres gigantesques sont préposés à la garde des palais, d'autres puissances y sont également représentées, que l'on peut identifier avec des génies. C'est le cas du génie-griffon - sur un corps d'homme se greffent une tête et des ailes d'oiseau de plus de deux mètres de hauteur, qui figure sur un relief monumental du palais royal du roi assyrien Assurnasirpal II à Nimrud et qui date du Ix ${ }^{e}$ siècle. Comme les

16. Parrot 1961, 83-86. 
autres génies, il semble occupé à une tâche bénéfique, selon toute apparence, qui doit être liée à une scène de rituel. Souvent leur est associé un arbre dont la stylisation a été poussée à son plus haut degré et qui est objet de vénération. Ils tiennent souvent à la main une situle (récipient rituel) et une pomme de pin destinées à des rites de purification. André Parrot ${ }^{17}$ en propose l'interprétation suivante : le génie capte sur l'arbre le fluide sacré qui émane de son tronc et de ses feuilles et en plongeant ensuite la pomme de pin dans la situle, il transforme le liquide qu'il contient en eau bénite. Avec elle le génie aspergera le roi pour le purifier sans doute, mais encore plus pour lui conférer une force supraterrestre, qui lui permettra de triompher des puissances mauvaises. Le même geste pourra se renouveler identique, à l'intention des acolytes du roi comme aussi des visiteurs admis à pénétrer dans la résidence du souverain.

Parmi les êtres hybrides protecteurs des hommes, il faut compter les hommessirènes barbus ou kulullû, peut-être inspirés des dugongs ${ }^{18}$ naguère attestés dans le golfe Persique. Dans l'art néo-assyrien, sur les reliefs monumentaux ou sous la forme de petites figurines, toujours représentés avec un torse, des bras et un visage humains tandis que le bas de leur corps se termine par une queue de poisson, ils servent de protection magique. Les sirènes féminines ou Kuliltu sont plus rares. Dès l'époque cassite, il existe plusieurs représentations d'hommes revêtus d'une peau de poisson complète (nageoires et queue comprises) dont la tête leur sert de couvre-chef; ils sont ainsi accoutrés de part et d'autre du malade pour conjurer Lamashtu dans certaines plaques magiques; on leur donne alors le rôle de prêtres exorcistes. Mais comme on les trouve également sculptés dans l'encadrement des portes des temples et palais assyriens, leur fonction est également de protéger magiquement les lieux par leur présence. C'est peut-être aussi cette figure qui a inspiré le personnage du sage Oannès de Bérose, puisque les Sept Sages qui vivaient avant le Déluge d'après la tradition babylonienne sont représentés parfois revêtus d'une peau de poisson. Ce ne sont pas des êtres composites, mais leur apparence en est très proche, puisque la peau de poisson est en fait probablement un vêtement rituel destiné à rendre l'homme caché dessous le plus semblable possible à un poisson.

Certains personnages en dehors des dieux sont détenteurs de savoir et ils initient les hommes à des découvertes capitales. Parmi les créatures composites, c'est Oannès qui joue ce rôle, comme le rapporte Bérose, « un lointain descendant des antiques lettrés de Mésopotamie » pour reprendre la présentation que Jean Bottéro

17. Parrot 1961, 70-71.

18. Cf. l'article "poissons» de Brigitte Lion dans Joannès et al. 2001. Précisons que les dugongs sont des mammiferes siréniens qui vivent dans l'océan Indien et peuvent atteindre trois mètres de long; ils se nourrissent de végétaux aquatiques. 
fait de ce personnage dans son ouvrage intitulé Lorsque les dieux faisaient l'homme. Ce Bérose était

attaché au Temple de Bêl [Marduk], à Babylone, et, vers 300 avant notre ère, [... s'était donné pour tâche de mettre en grec, dans ses Babyloniaca notamment, les principales traditions de son pays sur les origines du monde et sa plus vieille «histoire » ${ }^{19}$.

Nous n'avons conservé de son œuvre qu'un certain nombre de fragments rapportés par des auteurs anciens. Nous apprenons par le premier de ces fragments ${ }^{20}$ qu'Oannès, une créature mythique, amphibie, mi-homme, mi-poisson, serait sortie de la mer Rouge pour révéler aux hommes (de Mésopotamie) l'ensemble des connaissances qui fondent la civilisation. Cela se serait passé sous le règne d'Aloros, le premier des rois connus, qui a pour nom Alulim dans la Liste royale sumérienne ${ }^{21}$. Bérose fait le portrait suivant d'Oannès: son corps est celui d'un poisson, mais à côté de sa queue de poisson ont poussé deux pieds d'homme et sous sa tête de poisson, se trouve une autre tête (humaine); il était doté d'une voix humaine. Qu'a-t-il donc enseigné aux hommes au milieu desquels il vécut (le jour il allait sur terre, au coucher du soleil il plongeait dans la mer pour y rester toute la nuit) ? Bérose fait l'inventaire des connaissances innombrables que ce sage leur a transmises: la lecture et l'écriture, les mathématiques, la géométrie, la jurisprudence, l'expérience de toutes les techniques possibles; comment fonder un temple, une ville, comment cultiver des céréales et procéder pour la récolte des fruits; bref, tout ce qui constitue la vie civilisée. Puis Bérose présente une énumération de tous les «monstres» qu'Oannès a fait connaître aux hommes.

C'était au temps où tout était eau et ténèbres: on rencontrait alors entre autres des créatures composites comme les hommes à deux ou quatre ailes; des chiens à quatre corps et à queue de poisson; des centaures mi-hommes, mi-chevaux; des hommes à pattes et cornes de chèvre, à arrière-train ou simplement à queue de cheval; des taureaux à tête humaine; des chevaux à tête de chien; des êtres à tête et corps de cheval et à queue de poisson; des créatures rassemblant des parties de toutes sortes de bêtes sauvages. De toutes ces créatures et de bien d'autres, il y avait des représentations dans le temple de Bêl. Mais aucune des fonctions de ces êtres étonnants n'est précisée. On devine seulement l'originalité de la décoration d'un temple pareillement orné et quelle impression d'effroi et de respect il devait imposer aux fidèles de Bêl... et d'admiration pour les artistes qui les ont sculptés en leur donnant cet aspect si effrayant.

19. Bottéro 1989, 198-199.

20. TLG-E, fgt 1 a, 1. 27-70.

21. Pour un aperçu de cette liste des rois antédiluviens, cf. Burstein 1978. 
D'autres êtres composites partagent avec des monstres qui ne le sont pas la fonction suivante: la mise à l'épreuve de personnages par leur nature invincible que ce soit en raison de leur taille gigantesque, de leur action violente ou de leur pouvoir belliqueux et mortifère. Citons d'abord les plus célèbres, ceux qui, dans l'Épopée de la Création ou Enûma Elish ${ }^{22}$, qui date du dernier quart du II millénaire, vers 1125 environ, constituent le cortège de Tiamat, partie en guerre contre Marduk, munie de onze armes irrésistibles qu'elle a elle-même enfantées :

Elle mit au monde des Dragons-géants

Aux dents pointues,

Aux crocs impitoyables,

Dont elle emplit le corps

De venin en guise de sang;

Et des Léviathans féroces,

Qu'elle revêtit d'épouvante,

Et chargea d'Éclat-surnaturel,

les assimilant ainsi à des dieux:

«Qui les voit

Tombe en défaillance!

Et qu'une fois lancés (dit-elle),

Ils ne reculent jamais!»

Elle suscita encore des Hydres,

Des Dragons-formidables, des Monstres-marins,

Des Lions-colossaux,

des Molosses-enragés, des Hommes-scorpions,

Des Monstres-agressifs,

Des Hommes-Poissons, des Bisons-gigantesques;

Tous brandissant des armes impitoyables

Et sans peur au combat,

Leurs pouvoirs-délégués, démesurés,

Et eux, irrésistibles!

En vérité, ces onze-là,

C'est bien tels qu'elle les fit $^{23}$ !

Dans ce récit, Marduk se présente au combat en champion des dieux et sa victoire sur les monstres terrifiants envoyés par Tiamat fonde sa légitimité de roi des dieux, selon cette épopée. Pour que Marduk accède à la prééminence absolue sur les dieux, le monde et les hommes, il est indispensable que ses adversaires qui vont

22. L'Épopée de la Création ou Enûma Elish date du dernier quart du $\mathrm{II}^{\mathrm{e}}$ millénaire, vers 1125 environ.

23. Nous citons la traduction du « refrain » de cette épopée faite par J. Bottéro dans Bottéro 1989, 610611. 
mettre à l'épreuve sa puissance, sa combativité soient particulièrement féroces et terrifiques : l'étrangeté des créatures composites contribue ici à montrer comment Tiamat comptait déstabiliser son adversaire.

Mentionnons ensuite Ishtar (Inanna en sumérien), déesse de l'amour et de la guerre, dépitée d'être éconduite par Gilgamesh auquel elle s'était offerte ${ }^{24}$ : elle a envoyé contre ce dernier le Taureau-céleste, un taureau ailé qu'elle obtint de son père Anu (An en sumérien) afin de détruire, par vengeance, Uruk, la cité dont Gilgamesh est le roi. Mais Gilgamesh parvient à mettre à mort la terrifiante créature ailée et sort vainqueur de cette épreuve.

Citons encore les hommes-scorpions qui apparaissent également dans l'Épopée de Gilgamesh, mais ils forment un seul couple, mâle et femelle, et le premier joue le rôle principal : c'est lui qui interroge le héros Gilgamesh sur les raisons de sa venue jusqu'au défilé des Monts-Jumeaux, profond et obscur, que le Soleil empruntait chaque jour pour venir éclairer le monde. C'est précisément ce passage que contrôle le couple d'hommes-scorpions, apostés là pour empêcher quiconque de l'emprunter. Il faut dire que ces fabuleux gardiens suscitent une peur nonpareille à quiconque les aperçoit:

\author{
Des Hommes-scorpions \\ Défendaient leur entrée : \\ Il en émanait un effroi terrifique: \\ Leur seule vue, c'était la Mort! \\ Leur effrayant Éclat - surnaturel \\ Recouvrait ces Montagnes; \\ Ils n'étaient là que pour garder \\ L'itinéraire du Soleil! \\ Dès qu'il les aperçut, \\ La peur et l'épouvante \\ Couvrirent le visage de Gilgamesh. \\ Mais, prenant son parti, \\ Il s'approcha à leur rencontre ${ }^{25}$.
}

Ces créatures effrayantes jouent donc le rôle de gardiens et leur nature et leur stature sont telles qu'on imagine difficilement comment franchir le passage qu'elles contrôlent sans leur accord. Mais, de ce fait, elles mettent le héros à l'épreuve, héros qui doit rassembler toutes ses forces et son courage pour oser engager la discussion avec elles. Il n'y aura pas besoin de combat pour les tuer et s'ouvrir la voie, comme

24. Gilgamesh, en effet, jette au visage d'Ishtar la liste de ses amours qui ont toutes mal tourné pour ses amants, comme c'est raconté dans l'Épopée de Gilgamesh (Bottéro 1992, 271-273).

25. Épopée de Gilgamesh, tablette IX, II, vers 5-12 (Bottéro 1992, 158). 
ce fut le cas quand Gilgamesh fit face au Taureau-géant descendu du ciel, au gardien de la forêt des Cèdres, le fameux Humbaba et aux lions aux passes des Montagnes. La franchise et le désespoir du héros convainquent à eux seuls les deux gardiens surnaturels. C'est que Gilgamesh, désespéré par la mort de son ami Enkidu, a peur maintenant de mourir à son tour. Aussi, leur explique-t-il, lui est-il indispensable d'emprunter ce défilé des Monts-Jumeaux afin de rejoindre Utanapishti, héros rescapé du Déluge qui, en raison de sa piété, obtint l'immortalité et auprès duquel il compte découvrir le moyen d'éviter la mort, afin de garder la vie, sans $\mathrm{fin}^{26}$. Le héros surmonte l'épreuve par sa sincérité. Et les Hommes-scorpions finissent par lui donner des conseils pour trouver son chemin qui sera extrêmement long et difficile à parcourir: d'opposants, ils deviennent les adjuvants du héros.

Les créatures composites de l'ancienne Mésopotamie peuvent donc remplir divers rôles, notamment la garde de lieux sacrés, la protection (magique) des hommes; leurs connaissances font de certains d'entre eux des sages qui initient les hommes à la vie civilisée, ou plus simplement ils fournissent une aide au héros ; en revanche, certains tirent de leur caractère effrayant leur vocation d'opposant aux héros et mettent ces derniers à l'épreuve, tandis que d'autres épouvantent par leur simple présence et suscitent le respect notamment lorsqu'ils décorent temples et palais. Ces créatures hybrides fascinent par leur aspect étonnant et le souci de leur créateur est peut-être de susciter simplement un plaisir esthétique notamment dans les textes plus tardifs et la décoration de sceaux-cylindres.

\section{Comparaison entre êtres hybrides de Mésopotamie et de Grèce}

Nous pouvons remarquer, en premier lieu, en regardant la reproduction des principales figures d'êtres hybrides mésopotamiens dans les annexes de ce travail et le tableau intitulé «Les créatures composites grecques: aide-mémoire " ${ }^{27}$, que les animaux entrant en composition sont les mêmes en Mésopotamie et en Grèce, aux exceptions suivantes près : le coq, le sanglier et la biche pour la Grèce, l'âne, le scorpion, le bouquetin et le mouflon pour la Mésopotamie. Nous ne parlons pas des êtres qui, après une métamorphose sanctionnant une faute ou bêtise de leur part, se trouvent être des hybrides, comme le roi Midas avec ses oreilles d'âne. C'est pourquoi nous réservons l'âne au domaine de la Mésopotamie.

Pour les procédés de composition, les deux plus courants sont identiques:

- la juxtaposition d'éléments animaux (l'hippalektryon, les centaures...), ou d'éléments animaux et humains (tritons, sirènes, centaures, Minotaure...), mais en

26. Épopée de Gilgamesh, III, vers 5 (Bottéro 1992, 159).

27. Cf. Annexes I \& II. 
Grèce l'association d'un élément inanimé n'est pas exploité - rappelons qu'il l'est peu en Mésopotamie;

- l'adjonction d'éléments appartenant à une autre espèce qui confèrent à la créature le même cachet d'irréalité: cornes, sabots, serres, queue, ailes sont ainsi ajoutés à plusieurs personnages. La Gorgone, la Sphinge, Cerbère sont quelques exemples de ce procédé.

Certains de ces êtres hybrides mésopotamiens semblent avoir leur décalque du point de vue morphologique en Grèce, comme les hommes-poissons et les femmespoissons qu'on peut rapprocher des Sirènes et Tritons, des Telchines et de Dercéto, les êtres à tête et corps de cheval et à queue de poisson (dont Oannès a appris l'existence aux hommes) des ichthyocentaures, les hommes à pattes et cornes de chèvre (évoqués également par Oannès, dans le récit de Bérose) des Satyres et de Pan; les centaures qui perdent leurs ailes (un condensé du lion-centaure - moins le lion et du centaure - moins les ailes?), l'homme-taureau est à rapprocher du Minotaure, et le griffon est le même dans les deux civilisations (griffon qui, toutefois, trouve probablement son origine en Syrie au iI ${ }^{\mathrm{e}}$ millénaire et qui est connu dans l'ensemble du Proche-Orient, y compris en Mésopotamie et en Grèce au xiv siècle).

Ensuite, les rôles attribués aux créatures composites de Mésopotamie recoupent ceux qui sont dévolus aux êtres hybrides grecs: qu'on se rapporte à notre tableau «aide-mémoire» pour s'en persuader.

Aux gardiens Talos, Delphyné, Cerbère, Griffon en Grèce correspondent Humbaba, les hommes-scorpions, les taureaux et lions ailés androcéphales en Mésopotamie.

En ce qui concerne les protecteurs et adjuvants, les Grecs en ont inventé comme les Mésopotamiens. Citons Pégase grâce auquel Bellérophon tue Méduse; ou bien les dragons ailés attelés au char de Triptolème, offerts par Déméter pour semer des grains de blé en parcourant le monde...

Éprouver la valeur d'un héros: c'est peut-être dans ce domaine que les hybrides jouent le plus de rôle en Grèce à la différence des créatures composites de Mésopotamie qui s'illustrent autant dans la protection des hommes : on rapprochera les Hommes-scorpions et Hommes-poissons de la série des Onze, les monstres tués par Gilgamesh (le Taureau céleste, Humbaba aux griffes de lion) des animaux composites qu'affrontent Héraclès, Jason... ou de Typhon que doit affronter Zeus.

Éduquer les hommes et leur livrer des connaissances est le propre d'Oannès et les centaures Chiron et Pholos ou Silène et Marsyas ont une mission de même nature. Et la comparaison avec Oannès est encore plus intéressante avec les Telchines qui sont des détenteurs de savoirs amphibies, mi-hommes, mi-poissons. Ce sont eux qui ont élevé Poséidon; ils ont par ailleurs le pouvoir magique de faire tomber pluie, neige et grêle ; et l'on dit qu'ils ont inventé de nombreux arts, en particulier l'art de sculpter les statues des dieux. 
C'est encore une fonction commune aux créatures composites de Mésopotamie et de Grèce que d'orner par leur aspect fantastique les édifices dédiés aux dieux ou de petits objets en pierre fine, en ivoire... dans la confection desquels les artistes déploient toute leur habileté: les griffons communs aux deux civilisations jouent ce rôle, tout comme l'Hippalektryon ou bien l'Ichthyocentaure.

Quant à Imdugud, le démon météorologique du vent et des tempêtes, il peut avoir inspiré la figure des Harpyes : ils ont en commun de susciter l'orage par un battement d'ailes pour le premier et de personnifier les vents violents pour les secondes qui sont également ailées. De plus, leur nom (Bourrasque, Vole-vite et Obscure) fait irrésistiblement penser au ciel traversé par une nuée d'orage.

Enfin, les créatures dont une partie du corps est celle d'un serpent sont attribuées à diverses divinités en Mésopotamie et elles ont globalement un rôle protecteur. Ceci est peut-être à rapprocher des êtres de même type en Grèce, tels Cécrops et Erichthonios, rois mythiques d'Athènes et détenteurs de savoir qui, à ce titre, ont un rôle positif à jouer auprès de leur peuple.

\section{Une spécificité de certaines créatures composites grecques : un élément métallique}

Nous pouvons observer que les Grecs se différencient des Mésopotamiens notamment sur le point suivant. Dans la composition des créatures hybrides grecques peut entrer une composante métallique et là réside une originalité du système de composition grec. Ainsi, nous rencontrons les créatures auxquelles Héraclès doit faire face : la Biche cérynite aux sabots d'airain et à la ramure d'or; les Oiseaux du lac Stymphale qui, dans certaines variantes du mythe, se servent de leurs plumes à pointe de bronze comme de flèches meurtrières ; les êtres effrayants que Jason et les Argonautes doivent affronter : les taureaux aux sabots d'airain dont la gueule souffle du feu; Talos, homme ou taureau de bronze dont le corps est parcouru d'une veine unique, bouchée à l'une de ses extrémités par un clou en bronze ; et l'animal pour lequel le héros et ses compagnons subissent tant d'épreuves : le bélier à la toison d'or ; n'oublions pas l'affreuse Gorgone, Méduse, à laquelle Persée parvient à trancher la tête: sa composition change légèrement d'une variante du mythe à l'autre et l'on retiendra que son corps est celui d'une femme, sa tête est hérissée d'anneaux écailleux de serpents, sa bouche flanquée de deux grosses défenses de sanglier, son cou recouvert d'écailles de dragon, ses mains sont en bronze et ses ailes en or.

Tous ces êtres hybrides, métalliques, ont la même fonction dans les récits mythologiques, la mise à l'épreuve du héros, parce qu'ils représentent en eux-mêmes ou bien un obstacle que les héros ne pourront franchir ou bien un objet qu'ils ne pourront conquérir sans déployer un trésor d'inventivité ou une force surhumaine, sans l'aide de la magie ou des dieux. Quand il s'agit du bronze, la créature hybride semble 
invulnérable ou dangereuse; quand il s'agit de l'or, les créatures composites sont plus souvent des objets de quête et de conquête.

Il faudrait étudier quels liens ces créatures peuvent entretenir avec les servantes robots en or d'Héphaïstos dans l'Iliade, XVIII, 417-420 et les hommes du mythe des races hésiodique, en dehors de la valeur symbolique accordée aux métaux. (Et de ce fait reposer la question de l'origine du mythe de la statue composée de plusieurs métaux chez Daniel et de l'arbre de la même apparence en Iran ancien) ${ }^{28}$.

\section{Détour par le monde hittite}

À propos des êtres hybrides métalliques de Grèce, faisons un détour par la civilisation hittite où ont également été créés des divinités et des monstres au corps composite qui apparaissent dans les décors des édifices ou sous différentes formes artistiques. Certaines de ces créatures sont propres aux Hittites, d'autres manifestement inspirées par la Mésopotamie.

Concernant la spécificité grecque qui consiste à mêler un élément métallique dans la composition d'un être hybride, deux rapprochements avec les créatures composites hittites sont intéressants, ceux que l'on peut établir avec le dieu-épée et avec le dieu-clou.

Du dieu-épée on peut trouver une représentation à Yazilikaya ${ }^{29}$, dans la chambre B du sanctuaire, sur le mur Est. Ce relief représente « une épée emblématique fichée verticalement dans le sol, le bouton fait d'une tête de dieu, la poignée de protomes de lions et de lions affrontés la tête en bas », explique K. Bittel dans son ouvrage sur l'art hittite ${ }^{30}$. Cette poignée zoomorphe du dieu-épée n'a pas d'exact équivalent, même si les archéologues ont pu retrouver dans le Sud-Est, à Lagash, des combinaisons du même type datant du début de la période dynastique et de plus récentes à Ras Shamra, sur la côte de la Syrie du Nord. Pour comprendre la signification de la présence du dieu-épée dans le temple rupestre, il faut préciser qu'au même endroit, précédant cette représentation, on trouve dans le roc un relief du roi Tudhaliya IV, conduit et protégé par le dieu Sarruma, et en face, les «douze dieux » ${ }^{31}$, personnages en marche, identiques les uns aux autres. Ces trois motifs n'entretiennent

aucun rapport pictural, ils ne sont liés qu'au niveau du contenu, dans la mesure où ils illustrent des thèmes complémentaires: les enfers dans le cas des douze dieux et

28. Cf. Dumas-Reungoat 2001, 354 sq.

29. Il s'agit du temple rupestre situé à $1,5 \mathrm{~km}$ au nord-est de Bogazköy (Hattusa) et qui date de 12501220.

30. Bittel 1976, 216.

31. Ces douze dieux ne constituent pas le panthéon des Hittites, mais des Hourrites. Le sculpteur devait les reproduire dans ce sanctuaire qui se trouvait tout proche de la capitale. 
du dieu-épée, épée du dieu Nergal ou de son équivalent local; dans le cas du Grand Roi adorant son dieu, le culte des morts ${ }^{32}$.

Le dieu-épée, hybride composé d'homme, de lion et de métal implicitement pour la lame de l'épée, offre donc un mélange pour le moins surprenant, dans lequel, comme en Grèce, le métal a un rôle à jouer. Mais on ne peut pas pour autant lui attribuer une fonction semblable à celle des hybrides métalliques grecs qui étaient manifestement conçus pour éprouver la valeur des héros (l'accent étant mis sur l'invulnérabilité impliquée par le métal). Toutefois, on peut supposer que le dieu-épée devait inspirer une certaine frayeur, dans la mesure où il s'apparente à Nergal, dieu des enfers, maittre du monde souterrain, dans la mythologie mésopotamienne. Ce dieu chthonien présente la particularité d'établir un lien entre le monde souterrain et le monde terrestre. Le plus souvent c'est un dieu destructeur qui provoque des épidémies mortelles ou des incendies, entre autres fléaux. Et l'une de ses armes est l'épée. On peut le déduire notamment de textes qui montrent qu'on lui offrait en ex-voto des épées ${ }^{33}$ (appelées namsaru en akkadien) et la lexicographie assyro-babylonienne a ensuite établi une relation privilégiée entre le dieu et l'objet en fournissant une équivalence ${ }^{34}$ entre namsaru et U. GUR qui est un idéogramme sumérien, particulièrement en usage au $\mathrm{I}^{\mathrm{er}}$ millénaire, désignant le dieu Nergal (quand il est précédé du déterminatif des noms divins).

Quant au second rapprochement, il concerne une figure divine particulière: un dieu à haute tiare conique dont la partie inférieure du corps prend la forme d'un clou. Nous n'avons trouvé aucune indication particulière concernant la statuette du II $^{\mathrm{e}}$ millénaire le représentant ${ }^{35}$. Faut-il rapprocher ce dieu-clou du dieu-épée, la pointe du clou s'apparentant alors à la lame de l'épée, bien qu'il ne présente aucune composante animale (comme le lion dans le cas du dieu-épée) ? Ou bien faut-il voir dans cette figure l'influence de la civilisation mésopotamienne, civilisation de l'écriture cunéiforme, puisque l'on sait que les Hittites ont transcrit leur langue en hiéroglyphes hittites et en cunéiformes hérités de Mésopotamie?

Par ailleurs, dans l'art hittite, figurent nombre d'êtres hybrides dans l'élaboration desquels les modèles mésopotamiens plus anciens ont dû jouer un rôle, comme le note $\mathrm{K}$. Bittel, notamment à propos de l'aigle bicéphale souvent représenté dans la civilisation hittite et dont l'introduction chez les Hittites date du XviII ${ }^{e}$ siècle. Il remarque que:

32. Bittel 1976, 216-217. K. Bittel précise que le dieu protecteur et le roi sont connus par ailleurs dans les empreintes de sceaux des Grands Rois : sur les parois du temple rupestre on en a donc simplement une représentation monumentale.

33. Cf. Joannès et al. 2001, article Nergal, 572-574.

34. C'est-à-dire que les deux mots, désignant d'une part l'épée et d'autre part Nergal, s'écrivaient grâce à la même combinaison de clous (combinaison n ${ }^{0} 417$ du Manuel d'épigraphie akkadienne de R. Labat et F. Malbran-Labat, 6 éd., Paris, Geuthner, 1988).

35. Pour une représentation de la statuette (d'origine inconnue), cf. Bittel 1976, 101, fig. 93. 
La combinaison aigle-lion, comme dans l'ancienne Mésopotamie, et celle de l'aigle et du mouflon, sont attestées chacune une fois dans la glyptique du XviII ${ }^{\mathrm{e}}$ siècle $^{36}$.

Parmi les êtres bicéphales, signalons la figure de la Chimère, que l'on retrouve en Grèce. Chez les Hittites, elle se présente comme une sphinge à deux têtes, l'une humaine, l'autre de lionne, son corps est celui d'un lion, sa queue est celle d'un serpent et son corps de lion est pourvu d'ailes. On trouve une représentation de cet animal fabuleux sur un relief d'orthostate en basalte datant du $\mathrm{IX}^{\mathrm{e}}$ siècle sur le Mur du Héraut à Karkemish ${ }^{37}$. De la même période, ou un peu avant, date un relief de la porte de la citadelle à Zincirli ${ }^{38}$ qui représente un démon à tête de lion, pourvu d'ailes. À cette période le nombre important d'êtres hybrides représentés montre que l'élément démonique exerçait une forte influence sur les idées d'alors ${ }^{39}$.

L'héritage de la Mésopotamie du Nord et des Hourrites se lit encore dans la représentation des griffons et des génies ailés à tête d'oiseau ou encore des êtres mihommes, mi-lions. L'art hittite propose également des Sphinx (à une seule tête) ou encore des hommes-taureaux (dont la tête est celle d'un homme barbu et le corps, à partir de la taille, celui d'un taureau) qui, le plus souvent, sont représentés en train de soutenir le soleil ailé ${ }^{40}$.

Signalons enfin une créature hybride tout à fait originale, mais qui n'a pas d'équivalent dans le monde grec: le dieu-montagne. Comme les Assyriens ${ }^{41}$, dès le XıII ${ }^{\mathrm{e}}$ siècle, les Hittites ont également mêlé dans leurs créations d'êtres hybrides une composante ni humaine, ni animale, mais qui n'est cette fois pas le bateau mais la montagne. Le dieu montagne est semblable à un homme pour la tête, le haut du corps et les pieds, tandis que pour le reste c'est une montagne. L'on rencontre, par exemple, une représentation de ce dieu-montagne liée au dieu Teshub, dieu de l'Orage du Ciel : ce dernier en effet est souvent représenté dans une position surélevée par rapport aux autres personnages qui l'entourent en étant placé sur des dieux-montagnes à forme en partie humaine ${ }^{42}$. On sait que pour les Hittites, les rocs et montagnes

36. Bittel 1976, 94 .

37. Karkemish est une ville située sur la rive droite de l'Euphrate, tout près de la frontière turco-syrienne, où la présence de rois hittites est attestée vers 1700 av. J.-C.

38. L'art figuratif de Zincirli voit ses créations les plus anciennes apparaître au néo-Hittite I (1000-980) ou au début du néo-Hittite II (950-850).

39. Cf. Bittel 1976, 266.

40. Le motif d'êtres hybrides placés sous le ciel et le soleil est fréquent dans la glyptique de Mitanni (1380-1340) et de la période assyrienne moyenne (1380-1030).

41. On trouve à Assur, dans le palais de Tukulti-Ninurta I, un dieu-montagne qui tient dans ses mains un vase jaillissant, symbole de la fertilité et de la prospérité du pays.

42. Pour une représentation du dieu-montagne, cf. par exemple Bittel 1976, 279, fig. 318 (il s'agit d'un relief d'orthostate, datant du $\mathrm{x}^{\mathrm{e}}$ siècle, sur lequel le dieu-montagne est entouré de deux génies à tête d'oiseau). 
renfermaient de puissantes forces occultes. À leurs yeux la montagne était donc un élément vivant par excellence. Aussi ne doit-on pas s'étonner peut-être de ces représentations qui tentent de mettre en images le caractère divin de la nature.

Ainsi, chez les Hittites, une forte influence de la Mésopotamie se fait sentir, ce qui n'empêche pas la création d'êtres hybrides propres au système religieux et mythologique hittite. Nous ne pouvons pas toujours connaitre les conditions dans lesquelles l'influence de l'art de l'ancienne Mésopotamie s'est exercée sur celui des Hittites, mais un document intéressant nous renseigne sur les échanges entre les deux royaumes au milieu du XIII ${ }^{e}$ siècle: on sait par une lettre envoyée par Hattusili III à Kâdasman-Enlil de Babylone que le roi hittite prie son homologue babylonien de lui fournir un sculpteur et que des artistes de Babylone étaient à l'occasion employés par la cour hittite du milieu du XIII ${ }^{e}$ siècle. Et nous ne savons pas toujours non plus comment les Grecs ont reçu les héritages de Mésopotamie, mais il est vraisemblable que les Hittites ont joué un rôle dans cette transmission.

Au terme de cette promenade parmi les créatures composites mésopotamiennes nous constatons que les êtres divins ou monstrueux composés d'éléments humains et animaux sont très nombreux et très divers dans l'art et la littérature du ProcheOrient ${ }^{43}$ et de la Grèce. Ces corps imaginaires sont construits, pour la plupart, à partir de procédés identiques et l'on peut assez sûrement leur attribuer un rôle précis. En ce domaine, comme dans beaucoup d'autres, la Mésopotamie a exercé une forte influence sur la pensée hittite, sur la pensée grecque ancienne (par l'intermédiaire des Hittites ou directement) et plus près de nous encore dans le temps. Lisons, en effet, les propos d'André Parrot, dans son ouvrage sur Assur ${ }^{44}$, au sujet d'une survivance étonnante du taureau lion androcéphale et ailé aposté à divers palais de Mésopotamie :

43. Nous avons choisi de présenter les hybrides de Mésopotamie et d'Anatolie, mais pas ceux de l'Égypte. En Égypte, en effet, nombre de dieux sont représentés sous une forme hybride, mêlant composantes humaines et animales (beaucoup possédaient un corps humain et une tête animale, pour d'autres c'était l'inverse), pourtant nous ne traitons pas de ces représentations parce qu'elles s'inscrivent dans un imaginaire qui n'obéit pas, nous semble-t-il, aux mêmes lois qu'en Grèce et qu'en Mésopotamie. C'est que, parmi les grands dieux égyptiens, aucun ne s'est manifesté sous une seule et unique apparence. Prenons l'exemple de Thot, qui pouvait se présenter sous la forme d'un homme, d'un ibis ou sous la forme d'un homme à tête d'ibis ou de babouin : pour les anciens égyptiens, «Le dieu était caché dans le ciel, invisible aux hommes vivants. Pour ne pas entamer son pouvoir, sa forme véritable - tout comme son nom véritable - demeurait secrète. Ses icônes, même si elles contenaient une part de sa substance et pour cela devaient être adorées, n'étaient que des intermédiaires, des traductions partielles à l'usage des hommes, des outils sacrés. Aussi n'y avait-il aucune raison pour qu'elles demeurent immuables ", explique Alain Blottière dans son Petit dictionnaire des dieux égyptiens (Blottière 2000, 7-15).

44. Parrot 1961, 207. 


\section{Créatures composites en Mésopotamie}

En levant les yeux, au tympan des portails, on aperçoit le Christ en majesté, environné du tétramorphe. Jamais l'Orient n'a été plus présent. Il est là, au-dessus de nos têtes, car ces quatre figures, cet homme, ce lion, ce taureau et cet aigle, la symbolique chrétienne les a reprises, sans y rien changer, à la symbolique mésopotamienne, qui connaissait déjà à Ur, bien avant Ézéchiel, et dès le III ${ }^{\mathrm{e}}$ millénaire, ces quatre éléments isolés que les Assyriens eurent les premiers l'audace de réunir en un seul être: le taureau androcéphale gardien de leurs palais. Incroyable permanence des thèmes par-delà les races, les civilisations, les religions, et à travers le déroulement du temps. Langage immuable de la création qui, pour exprimer l'inexprimable, revient aux mêmes formules, aux mêmes figures ${ }^{45}$.

Christine Dumas-Reungoat

Université de Caen Basse-Normandie

\section{Références bibliographiques}

Bérose, Fragmenta Historicorum Graecorum, K. Müller (éd.) (1841-1870), FHG 2, Paris, Didot, fr. 1-25, p. 496-510 (édition utilisée dans le TLG-E).

Bittel K. (1976), Les Hittites, Paris, Gallimard (Univers des formes).

Black J., Green A. (1992), Gods, Demons and Symbols of Ancient Mesopotamia: An Illustrated Dictionary, Austin, University of Texas Press ( $4^{\mathrm{e}}$ édition en 2000).

Blotтì̀re A. (2000), Petit dictionnaire des dieux égyptiens, Cadeilhan, Zulma.

Вотте́ro J. (1972-1973), «Rapports sur les cours à l'École des hautes études», in L’Annuaire de la $I V$ section, Sciences historiques et philologiques (Antiquités assyro-babyloniennes), Paris, p. 93-131.

Bотте́ro J. (1989), Lorsque les dieux faisaient l’homme, Paris, Gallimard.

45. Comme exemple de tétramorphe nous pouvons regarder une reproduction du tympan du portail royal à Chartres, qui date du XII e siècle, donnée par A. Parrot (Parrot 1961, 207) pour illustrer son propos. Chaque animal est symboliquement associé à chacun des quatre évangélistes: l'homme symbolise Matthieu (qui a placé en tête de son évangile la généalogie humaine de Jésus); le lion, Marc (prédication de Jean-Baptiste dans le désert); le taureau, Luc (sacrifice de Zacharie); et l'aigle, Jean (prologue d'une grande élévation). C'est dans la mosaïque de l'abside de Santa Pudenziana, à Rome, qu'ils sont pour la première fois représentés sous la forme des quatre êtres vivants ailés inspirés d'Apocalypse, 4, 6-8. Les symboles des Évangélistes remontent à saint Jérôme (347419), docteur de l'Église qui interpréta les quatre êtres vivants d'Apocalypse, 4, 6-8 (tirés d'Ézéchiel, 1, 5-10) comme des images des Évangélistes. 
Bотте́ro J. (1992), L’Épopée de Gilgamesh, le grand homme qui ne voulait pas mourir, Paris, Gallimard (Aube des peuples).

Burstein S.M. (1978), "The Babyloniaca of Berossus», Sources and Monographs on the ancient near east, vol. 1 , fascicule 5 .

Dumas-Reungoat C. (2001), La Fin du Monde: enquête sur l'origine du mythe, Paris, Les Belles Lettres (Vérité des mythes).

Grimal P. (1951), Dictionnaire de la mythologie grecque et romaine, Paris, PUF.

JoAnnìs F. et al. (2001), Dictionnaire de la civilisation mésopotamienne, Paris, Robert Laffont (Bouquins).

Labat R. (1970), Les Religions du Proche-Orient asiatique, Paris, Fayard - Denoël.

Leibovici M. (1971), "Génies et démons en Babylonie », Sources orientales, 8, Génies, anges et démons: Égypte, Babylone, Israël, Islam..., p. 85-112.

Parrot A. (1960), Sumer, Paris, Gallimard (Univers des formes).

Parrot A. (1961), Assur, Paris, Gallimard (Univers des formes).

West M.L. (1960), Hesiod. Theogony, Oxford, Clarendon Press.

Et le lecteur consultera avec profit:

Padgett J.M. et al. (2003), The Centaur's Smile: The Human Animal in Early Greek Art, New Haven, Yale University Press. 


\section{Les créatures composites grecques : aide-mémoire}

\begin{tabular}{|c|c|c|}
\hline $\begin{array}{l}\text { Nom de la créature } \\
\text { composite }\end{array}$ & Composition & Fonction \\
\hline $\begin{array}{l}\text { Biche } \\
\text { aux pieds d'airain }\end{array}$ & Biche + métal (airain + cornes en or) & Éprouver un héros \\
\hline $\begin{array}{l}\text { Oiseaux du lac } \\
\text { Stymphale }\end{array}$ & Oiseau + métal (bronze) & Éprouver un héros \\
\hline Dercéto & Visage de femme + corps de poisson & $\begin{array}{l}\text { Déesse, mère de Sémira- } \\
\text { mis, victime d'Aphrodite }\end{array}$ \\
\hline Telchines & $\begin{array}{l}\text { Êtres amphibies, mi-marins, mi- } \\
\text { terrestres; bas du corps en forme de ser- } \\
\text { pent ou de poisson, ou bien pieds pal- } \\
\text { més }\end{array}$ & Détenteurs de savoirs \\
\hline Triton & $\begin{array}{l}\text { Dieu marin, corps semblable à celui } \\
\text { d'un homme + bas du corps terminé par } \\
\text { une queue de poisson }\end{array}$ & Protecteur des marins \\
\hline Sirènes & démons marins mi-femmes, mi-oiseaux & Éprouver un héros \\
\hline Minotaure & $\begin{array}{l}\text { Corps d'homme + tête et queue de tau- } \\
\text { reau }\end{array}$ & Éprouver un héros \\
\hline Centaures & $\begin{array}{l}\text { Buste d'homme }+ \text { reste du corps d'un } \\
\text { cheval (à l'époque classique, } 4 \text { pattes de } \\
\text { cheval }+2 \text { bras d'homme) }\end{array}$ & $\begin{array}{l}\text { Éprouver un héros; très } \\
\text { violents, liés aux légen- } \\
\text { des de viols et d'enlève- } \\
\text { ments }\end{array}$ \\
\hline Chiron et Pholos & $\begin{array}{l}\text { = centaures pour la morphologie, mais } \\
\text { de mœurs pacifiques }\end{array}$ & $\begin{array}{l}\text { Détenteurs de savoirs, } \\
\text { bienfaisants, hospitaliers }\end{array}$ \\
\hline Pégase & Cheval + ailes d'oiseau & Adjuvant du héros \\
\hline Delphyné & Dragon mi-femme, mi-serpent & Gardienne \\
\hline Échidna = « vipère » & $\begin{array}{l}\text { Monstre corps de femme }+ \text { bas du corps } \\
\text { en forme de serpent }\end{array}$ & $\begin{array}{l}\text { Met au monde des } \\
\text { monstres qui mettront } \\
\text { des héros à l'épreuve; } \\
\text { eut d'Héraclès Scythès, } \\
\text { qui donna son nom aux } \\
\text { Scythes }\end{array}$ \\
\hline Érichthonios & $\begin{array}{l}\text { Enfant à queue de serpent et / ou en- } \\
\text { touré de serpents }\end{array}$ & $\begin{array}{l}\text { Roi d'Athènes, détenteur } \\
\text { de savoirs+ Éprouver un } \\
\text { héros }\end{array}$ \\
\hline
\end{tabular}




\begin{tabular}{|c|c|c|}
\hline Cécrops & $\begin{array}{l}\text { Corps d'homme }+ \text { bas du corps } \\
\text { de serpent }\end{array}$ & $\begin{array}{l}\text { Premier roi mythique } \\
\text { d'Athènes + détenteur } \\
\text { de savoirs }\end{array}$ \\
\hline Satyres & $\begin{array}{l}\text { Corps d'homme (au sexe perpétuellement } \\
\text { dressé) + bas du corps de cheval ou de } \\
\text { bouc + queue de cheval + sabots de bouc; } \\
\text { petit à petit, leur figure est moins bestiale, } \\
\text { leurs membres inférieurs deviennent hu- } \\
\text { mains (pieds au lieu de sabots), mais ils } \\
\text { gardent de l'animal la queue }\end{array}$ & $\begin{array}{l}\text { Compagnons turbulents } \\
\text { et éducateurs de Dionysos }\end{array}$ \\
\hline Silène / Marsyas & Silène $=$ Satyre devenu vieux & $\begin{array}{l}\text { Marsyas = détenteur de } \\
\text { savoir }\end{array}$ \\
\hline Pan & $\begin{array}{l}\text { Dieu barbu au corps velu: torse et bras } \\
\text { d'homme + cornes, oreilles, pattes et sa- } \\
\text { bots de bouc }\end{array}$ & $\begin{array}{l}\text { Fécondité } \\
\text { Imaginaire alexandrin// } \\
\text { idylle rustique }\end{array}$ \\
\hline Cerbère & $\begin{array}{l}\text { Chien monstrueux à } 3 \text { ou } 50 \text { têtes }+ \text { cri- } \\
\text { nière ou queue constituée de serpents }\end{array}$ & $\begin{array}{l}\text { Gardien effrayant } \\
\text { Éprouver un héros }\end{array}$ \\
\hline $\begin{array}{l}\text { Harpies (Bourrasque, } \\
\text { Vole-Vite et Obscure) }\end{array}$ & Corps d'oiseau + visage humain & $\begin{array}{l}\text { Personnification des } \\
\text { vents violents }\end{array}$ \\
\hline $\begin{array}{l}\text { Grées (Enyo, } \\
\text { Péphrédo, Dino) }\end{array}$ & $\begin{array}{l}\text { Vieilles femmes au corps de cygne }+1 \\
\text { dent et } 1 \text { œil pour trois }\end{array}$ & Éprouver un héros \\
\hline Hippalektryon & $\begin{array}{l}\text { Corps, queue et pattes postérieures de } \\
\text { coq + tête et pattes antérieures de cheval }\end{array}$ & $\begin{array}{l}\text { Imaginaire pur } \\
\text { Fantastique décoratif }\end{array}$ \\
\hline $\begin{array}{l}\text { Ichthyocentaures ou } \\
\text { "Centaures- } \\
\text { poissons» }\end{array}$ & $\begin{array}{l}\text { Buste d'homme }+ \text { bas du corps d'un } \\
\text { poisson }+ \text { pattes de lion (souvent) }\end{array}$ & Fantastique décoratif \\
\hline Chimère & $\begin{array}{l}1 \text { tête de chèvre }+1 \text { tête de lion ou seule- } \\
\text { ment } 1 \text { tête de lion }+ \text { corps de chèvre } \\
+ \text { arrière-train de serpent }\end{array}$ & Éprouver un héros \\
\hline Gorgones & $\begin{array}{l}\text { Corps de femme + tête entourée de ser- } \\
\text { pents + grosses défenses de sanglier } \\
\text { + mains de bronze + ailes en or } \\
\text { + écailles de dragon sur le cou }\end{array}$ & Éprouver un héros \\
\hline Sphinx & $\begin{array}{l}\text { Monstre féminin au visage de femme } \\
+ \text { ou bien corps de lion + ailes d'aigle } \\
+ \text { parfois queue de dragon + seins de } \\
\text { femme, ou bien }+ \text { corps, poitrine et pat- } \\
\text { tes de lion + ailes d'oiseau de proie }\end{array}$ & Éprouver un héros \\
\hline Griffon & $\begin{array}{l}\text { Oiseau fabuleux : corps de lion }+ \text { tête } \\
\text { d'aigle }+ \text { très grandes ailes d'oiseau de } \\
\text { proie }\end{array}$ & $\begin{array}{l}\text { Gardien effrayant } \\
\text { Fantastique décoratif }\end{array}$ \\
\hline
\end{tabular}




\section{Créatures composites en Mésopotamie}
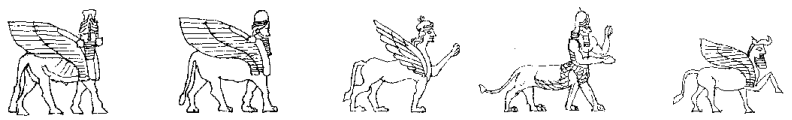

bulls and lions with human head

lion-centaur

Bull of Heaven

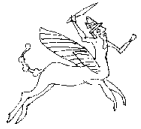

centaur
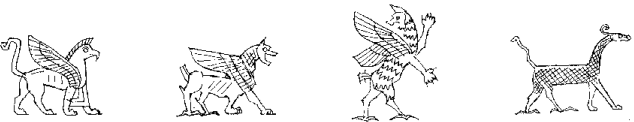

griffin

lion-dragon

$$
\begin{gathered}
\text { Asakku } \\
\text { (Asag)/Anzû́ } \\
\text { (Imdugud) }
\end{gathered}
$$

snake-dragon
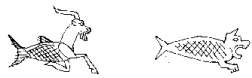

goat-fish

lion-fish
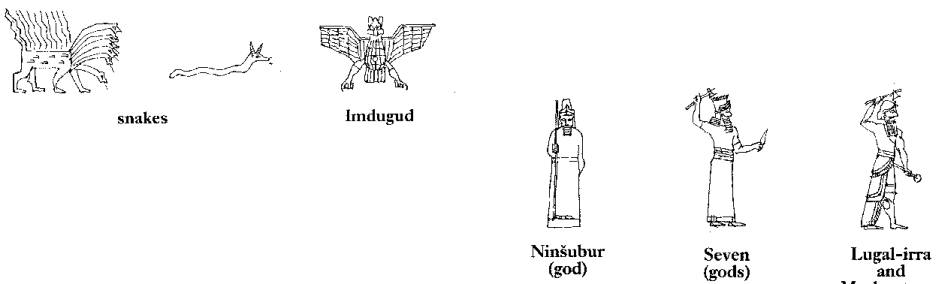

Meslamta-ea
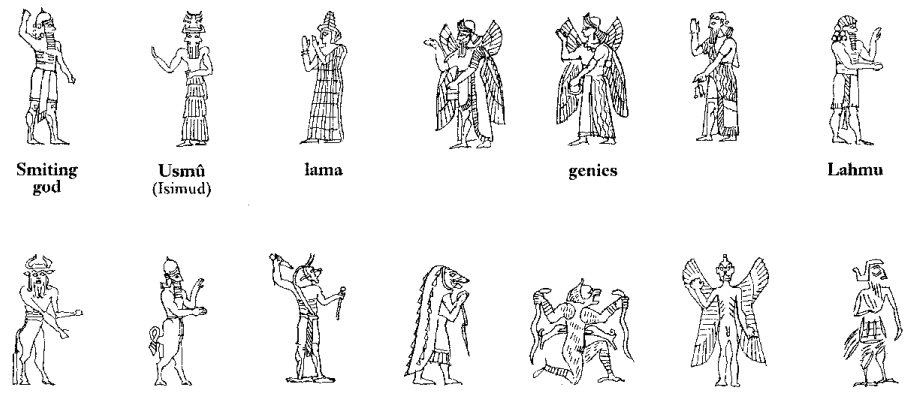

bull-man

lion-humanoid

lion-demon

La-tarāk

Lamaštu

Pazuzu

bird-man
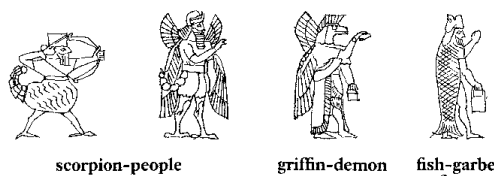

griffin-demon

fish-garbed
figure
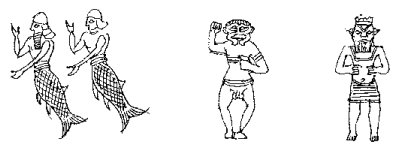

merman
and mermaid

Huwawa
(Humbaba)

Bes

Fig. 1 - Reproduction de la figure 53 " Demons, monsters and minor protective deities", in Black \& Green 1992, 64-65. 